\title{
FULLY DISCRETE SCHEMES FOR THE SCHRÖDINGER EQUATION. DISPERSIVE PROPERTIES
}

\author{
Liviu I. Ignat* \\ Departamento de Matemáticas, Universidad Autónoma de Madrid, \\ Crt. Colmenar Viejo, km. 15, Madrid, 28049, Spain \\ Institute of Mathematics of the Romanian Academy \\ P.O.Box 1-764, RO-014700 Bucharest, Romania
}

\begin{abstract}
We consider fully discrete schemes for the one dimensional linear Schrödinger equation and analyze whether the classical dispersive properties of the continuous model are presented in these approximations. In particular Strichartz estimates and the local smoothing of the numerical solutions are analyzed. Using a backward Euler approximation of the linear semigroup we introduce a convergent scheme for the nonlinear Schrödinger equation with nonlinearities which cannot be treated by energy methods.
\end{abstract}

\section{Introduction}

Let us consider the linear (LSE) Schrödinger equation

$$
\left\{\begin{array}{l}
i u_{t}+\Delta u=0, x \in \mathbb{R}^{d}, t \neq 0 \\
u(0, x)=\varphi(x), x \in \mathbb{R}^{d}
\end{array}\right.
$$

Its solution is given by $u(t)=S(t) \varphi$, where $S(t)=e^{i t \Delta}$ is the free Schrödinger operator. The linear semigroup $S(t)$ has two important properties, the conservation of the $L^{2}$-norm

$$
\|S(t) \varphi\|_{L^{2}\left(\mathbb{R}^{d}\right)}=\|\varphi\|_{L^{2}\left(\mathbb{R}^{d}\right)}, t \in \mathbb{R}
$$

and the dispersive estimate:

$$
|S(t) \varphi(x)|=|u(t, x)| \leq \frac{1}{(4 \pi|t|)^{d / 2}}\|\varphi\|_{L^{1}\left(\mathbb{R}^{d}\right)}, x \in \mathbb{R}^{d}, t \neq 0 .
$$

*email liviu.ignat@uam.es 
More refined space-time estimates known as the Strichartz inequalities show that, in addition to the decay of the solutions as $t \rightarrow \infty$, a gain of spatial integrability occurs. Improving the work of Strichartz [24], Ginibre and Velo[7] have proved that

$$
\|S(\cdot) \varphi\|_{L^{q}\left(\mathbb{R}, L^{r}\left(\mathbb{R}^{d}\right)\right)} \leq C(q, r)\|\varphi\|_{L^{2}(\mathbb{R})}
$$

for the so-called admissible pairs $(q, r): q \geq 2,2 \leq r<2 d /(d-2)$ and

$$
\frac{2}{q}=d\left(\frac{1}{2}-\frac{1}{r}\right),(q, r, d) \neq(2, \infty, 2) .
$$

The end-point case $q=2, r=2 d /(d-2)$ has been finally achieved by Keel and Tao 15. The extension to the inhomogeneous linear Schrödinger equation is due to Yajima 28, and Cazenave and Weissler 4].

These properties are not only relevant for a better understanding of the dynamics of the linear system but also to derive well-posedness results for inhomogeneous 24] and nonlinear Schrödinger equations. Typically the dispersive estimates are used when the energy methods fail to provide well posedness of the nonlinear problems. They were first applied by Ginibre and Velo for nonlinear problems with $H^{1}\left(\mathbb{R}^{d}\right)$ initial data 7 . In the case of $L^{2}\left(\mathbb{R}^{d}\right)$-initial data and nonlinearity $F(u)=|u|^{p-1} u, p<1+4 / d$, Tsutsumi[26], using estimates (3), has proved the well-posedness and global existence of solutions. The critical case has been analyzed by Cazenave and Weissler [5.

The Schrödinger equation has another remarkable property: the gain of one half space derivative [6, 16] in $L_{x, t}^{2}$ :

$$
\sup _{x_{0}, R} \frac{1}{R} \int_{B\left(x_{0}, R\right)} \int_{-\infty}^{\infty}\left|(-\Delta)^{1 / 4} e^{i t \Delta} \varphi\right|^{2} d t d x \leq C\|\varphi\|_{L^{2}\left(\mathbb{R}^{d}\right)}^{2} .
$$

It has been used in the study of the nonlinear Schrödinger equation with nonlinearities involving derivatives [17. Also, this type of local smoothing effect has been used [27] to prove the existence a.e. of $\lim _{t \rightarrow 0} u(x, t)$ for solutions of the Schrödinger equation with initial data in $H^{s}\left(\mathbb{R}^{d}\right), s>1 / 2$.

In this paper we analyze whether fully discrete schemes for the one dimensional LSE have dispersive properties similar to (2), (3) and (5), uniform with respect to the mesh sizes. The study of these dispersion properties for approximation of the linear semigroup $S(t)$ is relevant for introducing convergent schemes in the nonlinear context. Since the well-posedness of the nonlinear Schrödinger equations requires a fine use of the dispersion properties, the convergence of the numerical scheme for the nonlinear problem cannot be proven if these dispersion properties are not verified at the numerical level.

Estimates similar to (5) on discrete solutions will give sufficient conditions to guarantee their compactness and thus the convergence towards the solution of the nonlinear Schrödinger equation. Without such an estimate, despite the uniform boundedness of the discrete solutions in the space $l^{\infty}\left(k \mathbb{N}, l^{2}(h \mathbb{Z})\right) \cap$ $l_{l o c}^{q}\left(k \mathbb{N}, l^{r}(h \mathbb{Z})\right)$, one cannot pass to the limit in the nonlinear term. 
For the conservative semi-discretization of the Schrödinger equation, the lack of uniform dispersive estimates has been observed by Ignat and Zuazua 10,12 . In the one dimensional case, the symbol of the Laplacian, $\xi^{2}$, is replaced by a discrete one $\sin ^{2}(\xi / 2)$ which vanishes its first and second derivative at the points $\pm \pi$ and $\pm \pi / 2$ of the spectrum. By concentrating wave packets at these pathological points it is possible to prove the lack of any uniform estimate of the type $(2),(3)$ or $(5)$. For the semidiscrete Schrödinger equation we also refer to Ref. 21. In that paper the authors analyze the Schrödinger equation on the lattice $h \mathbb{Z}^{d}$ without concentrating on parameter $h$. They obtain Strichartz-like estimates in a class of exponents $q$ and $r$ larger than (4).

In Ref. [19, the author considers an approximation of the KdV equation based on the backward Euler approximation of the linear semigroup and proves space time estimates for that approximation. Here, we mean to give necessary and sufficient conditions to guarantee the existence, at the discrete level, of dispersive properties for the Schrödinger equation. The methods presented here also work in any dimension but we do not know whether the conditions we give in this paper are necessary. This is due to the fact that the construction of counterexamples is more complicated in the case of a higher dimension.

In Sec. 6 we exemplify our results by considering two numerical schemes: backward Euler and Crank-Nicolson. The first one introduces dissipation and has similar dispersion properties as in the continuous case. The second one is conservative and presents the same pathologies as the semidiscrete scheme we have discussed before: no local integrability property or local smoothing effect, being uniform with respect to the mesh size. This suggests that additional techniques based on artificial numerical viscosity (see Ref. [10] for a semidiscrete case) or a two-grid method [8] have to be used. In Sec. 10 we analyze the possible application of the last method to the Crank-Nicolson scheme. Using fine properties of number theory, in particular cyclotomic polynomials, we prove that any two-grid algorithm applied to the Crank-Nicolson scheme would not provide uniform $l^{1}(h \mathbb{Z})-l^{\infty}(h \mathbb{Z})$ estimates.

In Sec. 8 we introduce a numerical scheme for the nonlinear Schrödinger equation based on the backward Euler approximation of the linear semigroup and prove that its solutions remain uniformly bounded in the spaces where the well-posedness of the nonlinear problem is guaranteed 3 , 25]. We point out that this can be done by using any other scheme that approximates the linear Schrödinger semigroup and has an $l^{1}(h \mathbb{Z})-l^{\infty}(h \mathbb{Z})$ decay of solutions uniform with respect to the mesh size.

\section{Finite difference approximation}

In order to introduce the finite-difference approximation of the LSE, it will be necessary to first introduce some notations. The space $\mathbb{R} \times \mathbb{R}$ will be replaced by the lattice $\mathbb{Z} \times \mathbb{Z}$, and instead of functions $u(t, \cdot)$ depending on $t \in \mathbb{R}$, consideration will be given to sequences $U^{n}=\left(U_{j}^{n}\right)_{j \in \mathbb{Z}}$ for $n \in \mathbb{Z}$. For a mesh size $h>0$ and a time step $k>0, U_{j}^{n}$ is supposed to approximate $u(n k, j h) ; u(t, x)$ 
being a solution of the LSE. In the sequel we shall assume that Courant's number $\lambda=k / h^{2}$ is kept constant as $h, k \rightarrow 0$, and we shall consider a two-level difference scheme:

$$
\left\{\begin{array}{l}
U^{n+1}=A_{\lambda} U^{n}, n \geq 0, \\
U^{0}=\varphi .
\end{array}\right.
$$

We will be more precise on the type of estimates we are looking for. Let us consider $T>0, h \rightarrow 0$ and $n \in \mathbb{Z}$ such that $n k \rightarrow T$. We will establish necessary and sufficient conditions on the operator $A_{\lambda}$ to guarantee that

$$
\left\|U^{n}\right\|_{l^{q}(h \mathbb{Z})} \leq C\left(T, \lambda, q, q_{0}\right)\left\|U^{0}\right\|_{l^{q_{0}}(h \mathbb{Z})}
$$

for some $q_{0}<q$ with $C\left(T, \lambda, q, q_{0}\right)$ independent of $h$, and then also on $k$. Property (7) guarantees that the solutions of (6) gain integrability with respect to the initial data and that property is uniform with respect to the mesh size. Once scheme (6) satisfies (7) we prove more general estimates of the type:

$$
\|U\|_{l^{q}\left(k \mathbb{N}, l^{r}(h \mathbb{Z})\right)} \leq C(q, r, \lambda)\left\|U^{0}\right\|_{l^{2}(h \mathbb{Z})},
$$

uniformly on $k$ and $h$, related by $k / h^{2}=\lambda$.

Using (6) as a numerical scheme for the linear semigroup, we introduce an approximation for the inhomogeneous Schrödinger equation with null initial data:

$$
\left\{\begin{array}{l}
U^{n+1}=A_{\lambda} U^{n}+k f(n+1), \quad n \geq 0, \\
U^{0}=0,
\end{array}\right.
$$

where $f(n)_{n \geq 1}$ is an approximation of the inhomogeneous term. The difference equation (9) has an explicit solution, given by the discretized version of Duhamel's Principle:

$$
(\Lambda f)(n, \cdot)=k \sum_{j=0}^{n} S_{\lambda}(n-j) f(j, \cdot)
$$

with the convention $f(0) \equiv 0$. The same problem of uniform $l^{q}\left(k \mathbb{N}, l^{q}(h \mathbb{Z})\right)$ estimates for solutions of Eq. (9) will be studied in Sec. 7 .

The local smoothing property will be analyzed in Sec. 5. We introduce the discrete fractional derivatives on the lattice $h \mathbb{Z}$ by defining the fractional derivative of order $s$, as:

$$
\left(\left(-\Delta_{h}\right)^{s / 2} U\right)_{j}=\int_{-\pi / h}^{\pi / h}\left|\frac{2}{h} \sin \left(\frac{\xi h}{2}\right)\right|^{s} e^{i j \xi h} \mathcal{F}_{h}(U)(\xi) d \xi, j \in \mathbb{Z} .
$$

where $\mathcal{F}_{h}(U)$ is the discrete Fourier transform at the scale $h$ of the sequence $U$ :

$$
\mathcal{F}_{h}(U)(\xi)=h \sum_{j \in \mathbb{Z}} e^{-i j \xi h} U_{j}
$$


In Sec. 5 we obtain necessary and sufficient conditions in order to guarantee that the solutions of scheme (6) satisfy for some positive $s$

$$
k \sum_{n k \leq 1}\left[h \sum_{|j| h \leq 1}\left|\left(\left(-\Delta_{h}\right)^{s / 2} U^{n}\right)_{j}\right|^{2}\right] \leq C(s, \lambda)\left[h \sum_{j \in \mathbb{Z}}\left|U_{j}^{0}\right|^{2}\right]
$$

for some constant $C(s, \lambda)$, independent of $h$ and $k$. In fact, once (11) is satisfied the above left hand sums can be taken over any finite set of indices $n k \leq T$ and $|j| h \leq R$.

The Fourier analysis of the scheme (see Iserles 13, Ch. 13), usually done in the context of the stability, allows us to write the solution at the step $n \geq 0$ of scheme (6) as

$$
\widehat{U}^{n}(\xi)=a_{\lambda}^{n}(\xi) \widehat{\varphi}(\xi), \xi \in[-\pi, \pi],
$$

where $a_{\lambda}(\xi)$ is the quotient of two trigonometrical polynomials and $\widehat{U}=\mathcal{F}_{1}(U)$. The stability and consistency of the scheme show that the symbol $a_{\lambda}$ satisfies $\left|a_{\lambda}(\xi)\right| \leq 1$ for all $\xi \in[-\pi, \pi]$ and $a_{\lambda}(\xi) \sim 1-i \lambda \xi^{2}, \xi \sim 0$ (see Ref. [1, p. 259 for more details). It follows, since $a_{\lambda}$ is analytic, that one of the following conditions is satisfied, namely: $\left|a_{\lambda}(\xi)\right| \equiv 1, \xi \in[-\pi, \pi]$, or $\left|a_{\lambda}(\xi)\right|<1$ for all but a finite set of points. The first case corresponds to a conservative scheme; the second one to a dissipative scheme.

From now on, we write the symbol $a_{\lambda}$ in polar form $a_{\lambda}=m_{\lambda} \exp \left(i \psi_{\lambda}\right)$ and write the solution $U^{n}$ in the semigroup formulation $U^{n}=S_{\lambda}(n) \varphi$. Thus the consistency of the scheme implies that $m_{\lambda}(\xi) \sim 1$ and $\psi_{\lambda}(\xi) \sim-\lambda \xi^{2}$ as $\xi \sim 0$.

\section{Uniform $l^{\infty}$-decay rates}

The main result concerning the long time behaviour of the discrete solutions is given in the following Theorem:

Theorem 3.1. Let us assume that the symbol $a_{\lambda}$ has the following property

$$
m_{\lambda}\left(\xi_{0}\right)=1 \Rightarrow\left|\psi_{\lambda}^{\prime \prime}\left(\xi_{0}\right)\right|>0 \text { or } m_{\lambda}^{\prime \prime}\left(\xi_{0}\right) \neq 0 .
$$

Then for any $q \geq 2$ there is a positive constant $C(q, \lambda)$ such that

$$
\left\|S_{\lambda}(n) \varphi\right\|_{l^{q}(h \mathbb{Z})} \leq C(q, \lambda)(n k)^{\frac{1}{2}\left(\frac{1}{q}-\frac{1}{q^{\prime}}\right)}\|\varphi\|_{l^{q^{\prime}}(h \mathbb{Z})}
$$

holds for all $n \neq 0, h, k>0$.

This estimate is similar to the $L^{q^{\prime}}(\mathbb{R})-L^{q}(\mathbb{R})$ decay of the continuous Schrödinger semigroup obtained by interpolation between (1) and (2). Choosing a positive time $T$ and $k_{n} \rightarrow 0$ such that $n k_{n} \rightarrow T$ and taking the limit in (14) we obtain exactly the estimate for the continuous case.

We point out that with the same notations as in the one-dimensional case, a similar result can be stated in $\mathbb{R}^{d}$ if the symbol $a_{\lambda}$ satisfies:

$$
m_{\lambda}\left(\xi_{0}\right)=1 \Rightarrow \operatorname{rank}\left(H_{\psi_{\lambda}}\left(\xi_{0}\right)\right)=d \text { or } \xi H_{m_{\lambda}}\left(\xi_{0}\right) \xi^{t}<0, \forall \xi \in \mathbb{R}^{d},
$$


where $H_{m_{\lambda}}$ is the hessian matrix. However, we do not know if the condition is necessary.

Condition (13) essentially says that at the points $\xi_{0}$ where $m_{\lambda}$ equals one, either $\psi_{\lambda}^{\prime \prime}$ does not vanish or $m_{\lambda}(\xi) \sim 1+\left(\xi-\xi_{0}\right)^{2} m_{\lambda}^{\prime \prime}\left(\xi_{0}\right)$ as $\xi \sim \xi_{0}$. Also, at any point different from zero where $\psi_{\lambda}^{\prime \prime}$ vanishes the dissipative effect of $m_{\lambda}$ is present and vanishes the spurious effects introduced at that point by the scheme.

In the case $h=1$, an additional estimate holds. Using the fact that the discrete spaces $l^{p}(\mathbb{Z})$ are embedded, we also have $\|S(0) \varphi\|_{l^{q}(\mathbb{Z})} \leq\|\varphi\|_{l^{q^{\prime}}(\mathbb{Z})}$ and then for all $n \geq 0$ :

$$
\left\|S_{\lambda}(n) \varphi\right\|_{l^{q}(\mathbb{Z})} \leq C(q, \lambda)(1+n)^{\frac{1}{2}\left(\frac{1}{q}-\frac{1}{q^{\prime}}\right)}\|\varphi\|_{l^{q^{\prime}}(h \mathbb{Z})} .
$$

More generally, for any $n$ and $n_{1}$ positive the following holds

$$
\left\|S_{\lambda}(n) S_{\lambda}\left(n_{1}\right)^{*} \varphi\right\|_{l^{q}(\mathbb{Z})} \leq \frac{C(\lambda)}{1+\left|n-n_{1}\right|^{\frac{1}{2}\left(\frac{1}{q^{\prime}}-\frac{1}{q}\right)}}\|\varphi\|_{l^{q^{\prime}}(\mathbb{Z})},
$$

$S_{\lambda}\left(n_{1}\right)^{*}$ being the adjoint of $S_{\lambda}\left(n_{1}\right)$. Estimate (16) follows by (15) observing that $\left(S_{\lambda}\left(n_{1}\right)^{*} \varphi\right)^{\wedge}=m_{\lambda}^{n_{1}} \exp \left(-i n_{1} \psi_{\lambda}\right) \widehat{\varphi}$. The adjoint operator $S_{\lambda}(n)^{*}$ has another property that will be used later to establish Strichartz-like estimates: $S_{\lambda}(n) \varphi=\overline{S_{\lambda}(n)^{*} \bar{\varphi}}$, a property that is also fulfilled by the continuous Schrödinger semigroup.

Proof. We will consider the cases $q=2$ and $q=\infty$ the other following by interpolation of these two. The case $q=2$ easily follows by the stability of the scheme. For the second one we re-scale all the $l^{p}(h \mathbb{Z})$-norms, reducing the proof to the case $h=1$ :

$$
\frac{(n k)^{1 / 2}\left\|S_{\lambda}(n) \varphi\right\|_{l^{\infty}(h \mathbb{Z})}}{\|\varphi\|_{l^{1}(h \mathbb{Z})}}=\frac{(n k)^{1 / 2}\left\|S_{\lambda}(n) \varphi\right\|_{l^{\infty}(\mathbb{Z})}}{h\|\varphi\|_{l^{1}(\mathbb{Z})}}=\lambda^{1 / 2} n^{1 / 2} \frac{\left\|S_{\lambda}(n) \varphi\right\|_{l^{\infty}(\mathbb{Z})}}{\|\varphi\|_{l^{1}(\mathbb{Z})}}
$$

Now we prove that the right hand side remains bounded as long as $n$ varies in $\mathbb{Z} \backslash\{0\}$. Using representation (12) of the solutions, we obtain that $S_{\lambda}(n) \varphi=$ $K_{\lambda}^{n} * \varphi$, where the kernel $K_{\lambda}^{n}$ is given by

$$
K_{\lambda, j}^{n}=\frac{1}{2 \pi} \int_{-\pi}^{\pi} m_{\lambda}^{n}(\xi) e^{i n \psi_{\lambda}(\xi)} e^{i j \xi} d \xi, n \geq 0, j \in \mathbb{Z} .
$$

Young's inequality shows that $\left\|S_{\lambda}(n) \varphi\right\|_{l^{\infty}(\mathbb{Z})} \leq\left\|K_{\lambda}^{n}\right\|_{l^{\infty}(\mathbb{Z})}\|\varphi\|_{l^{1}(\mathbb{Z})}$, so, it is sufficient to prove that $K_{\lambda}^{n}$ satisfies

$$
\sup _{j \in \mathbb{Z}}\left|K_{\lambda, j}^{n}\right| \leq \frac{c_{\lambda}}{n^{1 / 2}}, n \in \mathbb{Z}, n \neq 0
$$

for some positive constant $c_{\lambda}$.

The function $a_{\lambda}$ being analytic the set $\Lambda$ where $m_{\lambda}$ equals one is either the whole interval $[-\pi, \pi]$, or consists of a finite number of points. We remark that $m_{\lambda}(\xi) \sim 1$ as $\xi \sim 0$ so, $\Lambda$ is nonempty. 
The first case corresponds to a conservative scheme and in view of (3.1), $\psi_{\lambda}^{\prime \prime}$ keeps constant sign. Thus by Van der Corput's Lemma (see Ref. [23], p. 332) we get for all $n \geq 1$ :

$$
\left|K_{\lambda, j}^{n}\right| \leq c\left(n \inf _{\xi \in[-\pi, \pi]}\left|\psi_{\lambda}^{\prime \prime}(\xi)\right|\right)^{1 / 2} \leq \frac{c_{\lambda}}{n^{1 / 2}}
$$

for all $n \geq 1$.

Let us analyze the case of $\Lambda$ consisting of a finite number of points. Its elements are isolated and we consider without loss of generality that $\Lambda$ has a single point, namely $\xi_{0}$. At this point, $m_{\lambda}$ has a local maximum and thus $m_{\lambda}^{\prime}\left(\xi_{0}\right)=0$ and $m_{\lambda}^{\prime \prime}\left(\xi_{0}\right) \leq 0$.

Let us first consider the case $m_{\lambda}^{\prime \prime}\left(\xi_{0}\right)<0$. Taylor's expansion of $m_{\lambda}$ at $\xi=\xi_{0}$ gives us

$$
m_{\lambda}(\xi)=1+\left(\xi-\xi_{0}\right)^{2} m_{\lambda}^{\prime \prime}\left(\xi_{0}\right)+O\left(\left|\xi-\xi_{0}\right|^{3}\right), \xi \sim \xi_{0}
$$

and shows the existence of a positive $\epsilon$ such that

$$
m_{\lambda}(\xi) \leq 1+\frac{\left(\xi-\xi_{0}\right)^{2} m^{\prime \prime}\left(\xi_{0}\right)}{2} \text { for all } \xi \in\left(\xi_{0}-\epsilon, \xi_{0}+\epsilon\right) .
$$

Thus, the kernel $K_{\lambda}^{n}$ satisfies:

$$
\begin{aligned}
\left|K_{\lambda, j}^{n}\right| & \leq \frac{1}{2 \pi} \int_{\xi_{0}-\epsilon}^{\xi_{0}+\epsilon} m_{\lambda}^{n}(\xi) d \xi+\frac{1}{2 \pi} \int_{[-\pi, \pi] \backslash\left(\xi_{0}-\epsilon, \xi_{0}+\epsilon\right)} m_{\lambda}^{n}(\xi) d \xi \\
& \leq \frac{1}{2 \pi} \int_{\xi_{0}-\epsilon}^{\xi_{0}+\epsilon}\left(1+\frac{\left(\xi-\xi_{0}\right)^{2} m_{\lambda}^{\prime \prime}\left(\xi_{0}\right)}{2}\right)^{n} d \xi+\left(\sup _{\xi \in[-\pi, \pi] \backslash\left(\xi_{0}-\epsilon, \xi_{0}+\epsilon\right)} m_{\lambda}(\xi)\right)^{n} \\
& \leq \frac{1}{2 \pi} \int_{\xi_{0}-\epsilon}^{\xi_{0}+\epsilon} \exp \left(\frac{n\left(\xi-\xi_{0}\right)^{2} m_{\lambda}^{\prime \prime}\left(\xi_{0}\right)}{2}\right) d \xi+\frac{c_{1, \lambda}(\epsilon)}{n^{1 / 2}} \\
& \leq \frac{c_{1}}{2 \pi\left(n\left|m_{\lambda}^{\prime \prime}\left(\xi_{0}\right)\right|\right)^{1 / 2}}+\frac{c_{1, \lambda}(\epsilon)}{n^{1 / 2}}=\frac{c_{\lambda}}{n^{1 / 2}} .
\end{aligned}
$$

It remains to analyze the case $\psi_{\lambda}^{\prime \prime}\left(\xi_{0}\right) \neq 0$. The continuity of $\psi_{\lambda}^{\prime \prime}$ at $\xi=\xi_{0}$ implies the existence of positive $\epsilon$ and $\delta$ such that

$$
\left|\psi_{\lambda}^{\prime \prime}(\xi)\right| \geq \frac{\delta}{2}, \text { for all } \xi \in\left(\xi_{0}-\epsilon, \xi_{0}+\epsilon\right)
$$

Outside of the interval $\left(\xi_{0}-\epsilon, \xi_{0}+\epsilon\right)$, the function $m_{\lambda}$ is strictly less than one and we have the rough estimate

$$
\left|\int_{-\pi}^{\pi} m_{\lambda}^{n}(\xi) e^{i n \psi_{\lambda}(\xi)} e^{i j \xi} d \xi\right| \leq\left(\sup _{\xi \in[-\pi, \pi]} m_{\lambda}\right)^{n} \leq \frac{c_{\lambda}}{n^{1 / 2}} .
$$

On the interval $\left(\xi_{0}-\epsilon, \xi_{0}+\epsilon\right)$ applying again Van der Corput's Lemma we get

$$
\left|\int_{\xi_{0}-\epsilon}^{\xi_{0}+\epsilon} e^{i n \psi_{\lambda}(\xi)} m_{\lambda}^{n}(\xi) e^{i j \xi} d \xi\right| \leq \frac{\left\|m_{\lambda}^{n}\right\|_{L^{\infty}\left(\left(\xi_{0}-\epsilon, \xi_{0}+\epsilon\right)\right)}}{\left(n_{\xi \in\left(\xi_{0}-\epsilon, \xi_{0}+\epsilon\right)}\left|\psi_{\lambda}^{\prime \prime}(\xi)\right|\right)^{1 / 2}}=\frac{c_{\lambda}}{n^{1 / 2}} .
$$




\section{Lack of uniform dispersive estimates}

In this section we prove that condition (13) imposed in the previous section is also necessary. If it fails we prove the lack of any uniform dispersive estimate. This means that there is no uniform decay of solutions as in (2) nor a space time estimate similar to (3).

Theorem 4.1. Let $q>q_{0} \geq 1$. Assume that $a_{\lambda}$ does not satisfy (13). Then for any $T>0$

$$
\lim _{\substack{h \rightarrow 0 \\ n k \rightarrow T}} \sup _{\varphi \in l^{q_{0}}(h \mathbb{Z})} \frac{\left\|S_{\lambda}(n) \varphi\right\|_{l^{q}(h \mathbb{Z})}}{\|\varphi\|_{l^{q_{0}}(h \mathbb{Z})}}=\infty
$$

and

$$
\lim _{\substack{h \rightarrow 0 \\ n k \rightarrow T}} \sup _{\varphi \in l^{q_{0}}(h \mathbb{Z})} \frac{\sum_{n k \leq T}\left\|S_{\lambda}(n) \varphi\right\|_{l^{q}(h \mathbb{Z})}}{\|\varphi\|_{l^{q_{0}}(h \mathbb{Z})}}=\infty .
$$

Remark 4.1. Let $I^{h, k}$ be a space-time interpolator, piecewise constant or linear. For any fixed $T>0$ the uniform boundedness principle guarantees the existence of a function $\varphi \in L^{2}(\mathbb{R})$, a sequence $h_{n} \rightarrow 0, n k_{n}=T$ and functions $\varphi_{h_{n}}$ such that $I^{h_{n}, k_{n}} S(0) \varphi_{h_{n}} \rightarrow \varphi$ in $L^{2}(\mathbb{R})$ and $\left\|I^{h_{n}, k_{n}} S(\cdot) \varphi_{h_{n}}\right\|_{L^{1}\left([0, T], L^{r}(\mathbb{R})\right)} \rightarrow \infty$.

We remark that a scaling argument as below

$$
\frac{\left\|S_{\lambda}(n) \varphi\right\|_{l^{q}(h \mathbb{Z})}}{\|\varphi\|_{l^{q_{0}}(h \mathbb{Z})}}=h^{\frac{1}{q}-\frac{1}{q_{0}}} \frac{\left\|S_{\lambda}(n) \varphi\right\|_{l^{q}(\mathbb{Z})}}{\|\varphi\|_{l^{q_{0}}(\mathbb{Z})}}
$$

reduces (17) to the following one:

$$
\lim _{N \rightarrow \infty} N^{\frac{1}{2 q_{0}}-\frac{1}{2 q}} \sup _{\varphi \in l^{q_{0}}(\mathbb{Z})} \frac{\left\|S_{\lambda}(N) \varphi\right\|_{l^{q}(\mathbb{Z})}}{\|\varphi\|_{l^{q_{0}}(\mathbb{Z})}}=\infty .
$$

By a similar argument, we obtain the following for the second estimate

$$
\frac{k \sum_{n k \leq T}\left\|S_{\lambda}(n) \varphi\right\|_{l^{q}(h \mathbb{Z})}}{\|\varphi\|_{l^{q_{0}}(h \mathbb{Z})}}=\frac{\lambda h^{2+\frac{1}{q}-\frac{1}{q_{0}}} \sum_{n k \leq T}\left\|S_{\lambda}(n) \varphi\right\|_{l^{q}(\mathbb{Z})}}{\|\varphi\|_{l^{q_{0}}(\mathbb{Z})}} .
$$

Denoting $N=T / k,(18)$ is reduced to the following one:

$$
\lim _{N \rightarrow \infty} N^{-1-\frac{1}{2}\left(\frac{1}{q}-\frac{1}{q_{0}}\right)} \sup _{\varphi \in l^{q_{0}}(\mathbb{Z})} \frac{\sum_{n \leq N}\left\|S_{\lambda}(n) \varphi\right\|_{l^{q}(\mathbb{Z})}}{\|\varphi\|_{l^{q_{0}}(\mathbb{Z})}}=\infty .
$$

To prove that condition (13) is necessary, we introduce the operators $T_{\lambda}(n)$ defined by

$$
\left(T_{\lambda}(n) \varphi\right)(x)=\int_{-\pi}^{\pi} m_{\lambda}^{n}(\xi) e^{i n \psi_{\lambda}(\xi)} e^{i x \xi} \widehat{\varphi}(\xi) d \xi
$$


We point out that these operators are in fact band-limited interpolators of the discrete operators $S_{\lambda}(n)$. Observe also, that once we have a $2 \pi$-periodic function $\widehat{\varphi}$ we can define both operators, the continuous and the discrete one.

The results of Magyar et al[18] (see also Plancherel and Polya 20]) on bandlimited functions show that the following inequality

$$
\frac{\left\|S_{\lambda}(n) \varphi\right\|_{l^{q}(\mathbb{Z})}}{\|\varphi\|_{l^{q_{0}}(\mathbb{Z})}} \geq c\left(\lambda, q, q_{0}\right) \frac{\left\|T_{\lambda}(n) \varphi\right\|_{L^{q}(\mathbb{R})}}{\|\varphi\|_{L^{q_{0}}(\mathbb{R})}}
$$

holds for any $q>q_{0} \geq 1$ and for all $2 \pi$-periodic functions $\widehat{\varphi}$. This reduces estimates (17) and (18) on the operator $S_{\lambda}(n)$ to similar ones on $T_{\lambda}(n)$.

The following lemma is the key point in the proof of Theorem 17.

Lemma 4.1. Let us consider a symbol $a_{\lambda}$ that does not verify (13). Then there exist two positive constants $c(\lambda)$ and $c_{1}(\lambda)$ such that for all $N$ sufficiently large, there exists a function $\varphi_{N}$ that satisfies

$$
\left\|\varphi_{N}\right\|_{L^{p}(\mathbb{R})} \simeq N^{1 / 3 p} \text { for all } p \geq 1
$$

and

$$
\left|\left(T_{\lambda}(n) \varphi_{N}\right)(x)\right| \geq c(\lambda)
$$

for all $n \leq c_{1}(\lambda) N$ and $\left|x+n \psi_{\lambda}^{\prime}\left(\xi_{0}\right)\right| \leq c_{1}(\lambda) N^{1 / 3}$.

Proof. The assumption on $a_{\lambda}$ shows the existence of a point $\xi_{0} \in[-\pi, \pi]$ such that $m_{\lambda}\left(\xi_{0}\right)=1, \psi_{\lambda}^{\prime \prime}\left(\xi_{0}\right)=0$ and $m_{\lambda}^{\prime \prime}\left(\xi_{0}\right)=0$. Also, at $\xi_{0}, m_{\lambda}^{\prime}\left(\xi_{0}\right)=0, m_{\lambda}$ having a local maximum.

Let us first fix a positive function $\widehat{\varphi}_{1}$ supported on $(-1,1)$ such that $\widehat{\varphi}_{1}>1$ on $(-1 / 2,1 / 2)$. For all positive $N$, we set the function $\varphi_{N}$ as:

$$
\widehat{\varphi}_{N}(\xi)=N^{1 / 3} \widehat{\varphi}_{1}\left(N^{1 / 3}\left(\xi-\xi_{0}\right)\right) \text {. }
$$

Observe that $\widehat{\varphi}_{N}$ is supported on the interval $\left(\xi_{0}-N^{-1 / 3}, \xi_{0}+N^{-1 / 3}\right)$. For any $p \geq 1$ classical properties of the Fourier transform guarantee that

$$
\left\|\varphi_{N}\right\|_{L^{p}(\mathbb{R})} \simeq N^{-1 / 3 p}
$$

The mean value theorem allows us to bound from below the magnitude of the oscillatory integral occurring in the definition of $T_{\lambda}(n) \varphi_{N}$ as follows:

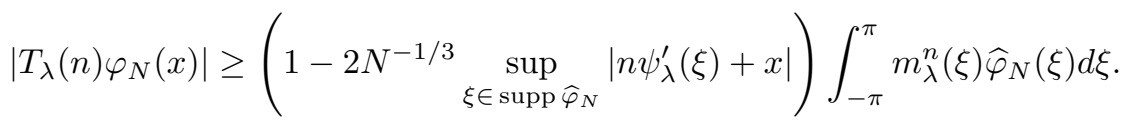

Using that the second derivative of $\psi_{\lambda}$ vanishes at $\xi=\xi_{0}$ we obtain the existence of a positive constant $c_{\lambda}$ such that

$$
\left|n \psi_{\lambda}^{\prime}(\xi)+x\right| \leq\left|n \psi_{\lambda}^{\prime}\left(\xi_{0}\right)+x\right|+n c_{\lambda}\left|\xi-\xi_{0}\right|^{2}, \xi \sim \xi_{0}
$$

In particular for all $\xi \in\left[\xi_{0}-N^{-1 / 3}, \xi_{0}+N^{-1 / 3}\right]$ the following holds

$$
\left|n \psi_{\lambda}^{\prime}(\xi+x)\right| \leq\left|n \psi_{\lambda}^{\prime}\left(\xi_{0}\right)+x\right|+n c_{\lambda} N^{-2 / 3} .
$$


Thus there exists a positive constant $c_{1}(\lambda)$ such that for all $x$ and $n$ satisfying $\left|x+n \psi_{\lambda}^{\prime}\left(\xi_{0}\right)\right| \leq c_{1}(\lambda) N^{1 / 3}$ and $n \leq c_{1}(\lambda) N$ :

$$
2 N^{-1 / 3} \sup _{\xi \in \operatorname{supp} \widehat{\varphi}_{N}}\left|n \psi_{\lambda}^{\prime}(\xi)+x\right| \leq \frac{1}{2}
$$

and

$$
\left|T_{\lambda}(n) \varphi_{N}(x)\right| \geq \frac{1}{2} \int_{-\pi}^{\pi} m_{\lambda}^{n}(\xi) \widehat{\varphi}_{N}(\xi) d \xi \geq \frac{N^{1 / 3}}{2} \int_{\xi_{0}-N^{-1 / 3} / 2}^{\xi_{0}+N^{-1 / 3} / 2} m_{\lambda}^{n}(\xi) d \xi .
$$

It remains to prove that for $N$ large enough the last term is uniformly bounded by below.

In the case $m_{\lambda} \equiv 1$ inequality (22) holds with $c(\lambda)=1 / 4$. Otherwise, we consider the smallest $k \geq 3$ such that $m_{\lambda}^{(k)}\left(\xi_{0}\right) \neq 0$. Thus for $\xi$ near $\xi_{0}$

$$
m_{\lambda}(\xi) \geq 1+\frac{m_{\lambda}^{(k)}\left(\xi_{0}\right)}{2}\left|\xi-\xi_{0}\right|^{k} .
$$

In view of this property, choosing $N$ sufficiently large we get

$$
\begin{gathered}
N^{1 / 3} \int_{\xi_{0}-N^{-1 / 3} / 2}^{\xi_{0}+N^{-1 / 3} / 2} m_{\lambda}^{n}(\xi) d \xi \geq N^{1 / 3} \int_{\xi_{0}-N^{-1 / 3} / 2}^{\xi_{0}+N^{-1 / 3} / 2}\left(1+\frac{m_{\lambda}^{(k)}\left(\xi_{0}\right)}{2}\left|\xi-\xi_{0}\right|^{k}\right)^{n} d \xi \\
\geq 2 N^{1 / 3} \int_{0}^{N^{-1 / 3} / 2} \exp \left(n m_{\lambda}^{(k)}\left(\xi_{0}\right) \frac{\xi^{k}}{2}\right) d \xi \\
=2 N^{1 / 3} n^{-1 / k} \int_{0}^{N^{-1 / 3} n^{1 / k}} \exp \left(m_{\lambda}^{(k)}\left(\xi_{0}\right) \frac{\xi^{k}}{2}\right) d \xi
\end{gathered}
$$

For all $n \leq c_{1}(\lambda) N$ we have that $N^{1 / 3} n^{-1 / k} \geq C(\lambda, k) N^{1 / 3-1 / k} \geq C(\lambda, k)$. Thus the the right hand term of (23) is uniformly bounded by below by a positive constant $c(\lambda)$, which proves (22) and finishes the proof of this Lemma.

In the following we apply the above Lemma to prove Theorem 4.1 .

Proof. of Theorem 4.1. We will prove (19), the other estimate (20) following by the same arguments, just using that (22) holds for all $n \leq c_{1}(\lambda) N$.

In view of (22), we will show that for $N$ large enough

$$
\sup _{\varphi} \frac{\left\|T_{\lambda}(N) \varphi\right\|_{L^{q}(\mathbb{R})}}{\|\varphi\|_{L^{q_{0}(\mathbb{R})}}} \geq c_{2}(\lambda) N^{1 / 3 q-1 / 3 q_{0}}
$$

holds for some positive constant $c_{2}(\lambda)$. Then by (21) we obtain (19) which finishes the proof.

First, let us choose $N_{1} \simeq N$ such that $N \leq c_{1}(\lambda) N_{1}$ with $c_{1}(\lambda)$ given by Lemma 4.1. Choosing $\varphi_{N_{1}}$ as in Lemma 4.1 we have that $\left\|\varphi_{N_{1}}\right\|_{L^{q_{0}(\mathbb{R})}} \simeq N^{1 / 3 q_{0}}$ and

$$
\left\|T_{\lambda}(N) \varphi_{N_{1}}\right\|_{L^{q}(\mathbb{R})} \geq\left|\left\{x:\left|x-N \psi_{\lambda}^{\prime}\left(\xi_{0}\right)\right| \leq c_{1}(\lambda) N^{1 / 3}\right\}\right|^{1 / q} c(\lambda) \geq c_{2}(\lambda) N^{1 / 3 q} .
$$


Finally we get that

$$
\frac{\left\|T_{\lambda}(N) \varphi_{N_{1}}\right\|_{L^{q}(\mathbb{R})}}{\left\|\varphi_{N_{1}}\right\|_{L^{q_{0}}}} \geq c_{2}(\lambda) N^{1 / 3 q-1 / 3 q_{0}}
$$

which proves (24) and finishes the proof.

\section{$5 \quad$ Local smoothing effect}

In this section we analyze the local smoothing effect that we discussed in the Introduction. In the following Theorem we obtain necessary and sufficient conditions such that this property holds uniformly with respect to the mesh size.

Theorem 5.1. There is a positive $s$ and a constant $C(s, \lambda)$ such that (11) holds for all $\varphi \in l^{2}(h \mathbb{Z})$ and $h>0$ if and only if the symbol $a_{\lambda}$ satisfies

$$
\xi_{0} \neq 0, \psi_{\lambda}^{\prime}\left(\xi_{0}\right)=0 \quad \Rightarrow \quad m_{\lambda}\left(\xi_{0}\right)<1 .
$$

Moreover if (25) holds then $s=1 / 2$ and

$$
\sup _{j \in \mathbb{Z}}\left[k \sum_{n \in \mathbb{Z}}\left|\left(\left(-\Delta_{h}\right)^{1 / 4} S_{\lambda}(n) \varphi\right)_{j}\right|^{2}\right] \leq C(\lambda) h \sum_{j \in \mathbb{Z}}\left|\varphi_{j}\right|^{2}
$$

holds for all $\varphi \in l^{2}(h \mathbb{Z})$ and all $h$.

Remark 5.1. Once (25) holds, a similar result can be stated for the inhomogeneous equation (9):

$$
k \sum_{n k \leq T} h \sum_{|j| h \leq R}\left|\left(\left(-\Delta_{h}\right)^{1 / 2} \Lambda f\right)(n, j)\right|^{2} \leq k \sum_{n k \leq T}\|f(n)\|_{l^{2}(h \mathbb{Z})}^{2} .
$$

Proof. We divide the proof in two steps. In the first one we prove that condition (25) implies estimate (26). Secondly we prove that condition (25) is necessary.

Step I. First by a scaling argument we obtain that

$$
\begin{aligned}
\frac{k \sum_{n \geq 0}\left|\left(\left(-\Delta_{h}\right)^{1 / 4} S_{\lambda}(n) \varphi\right)_{j}\right|^{2}}{h \sum_{j \in \mathbb{Z}}\left|\varphi_{j}\right|^{2}} & =\frac{k h^{-1} \sum_{n \geq 0}\left|\left(\left(-\Delta_{1}\right)^{1 / 4} S_{\lambda}(n) \varphi\right)_{j}\right|^{2}}{h \sum_{j \in \mathbb{Z}}\left|\varphi_{j}\right|^{2}} \\
& =\frac{\lambda \sum_{n \geq 0}\left|\left(\left(-\Delta_{1}\right)^{1 / 4} S_{\lambda}(n) \varphi\right)_{j}\right|^{2}}{\sum_{j \in \mathbb{Z}}\left|\varphi_{j}\right|^{2}},
\end{aligned}
$$

which reduces the proof to the case $h=1$.

Now we prove that under condition (25) the following estimate

$$
\sup _{j \in \mathbb{Z}} \sum_{n \geq 0}\left|\left(\left(-\Delta_{1}\right)^{1 / 4} S_{\lambda}(n) \varphi\right)_{j}\right|^{2} \leq c(\lambda) \sum_{j \in \mathbb{Z}}\left|\varphi_{j}\right|^{2}
$$


holds for all $\varphi \in l^{2}(\mathbb{Z})$ and for some positive constant $c(\lambda)$. The definition of $\left(-\Delta_{1}\right)^{1 / 4} S_{\lambda}(n) \varphi$ :

$$
\left(\left(-\Delta_{1}\right)^{1 / 4} S_{\lambda}(n) \varphi\right)_{j}=\int_{-\pi}^{\pi}|2 \sin (\xi / 2)|^{1 / 2} e^{i j \xi} e^{i n \psi_{\lambda}(\xi)} m_{\lambda}^{n}(\xi) \widehat{\varphi}(\xi) d \xi,
$$

and Plancherel's identity applied to the right hand term of (27)

$$
\sum_{j \in \mathbb{Z}}\left|\varphi_{j}\right|^{2}=\frac{1}{2 \pi} \int_{-\pi}^{\pi}|\widehat{\varphi}(\xi)|^{2} d \xi
$$

show that (27) is equivalent with the following estimate:

$$
\sup _{j \in \mathbb{Z}} \sum_{n \geq 0}\left|\left(S_{\lambda}(n) \varphi\right)_{j}\right|^{2} \leq c(\lambda) \int_{-\pi}^{\pi} \frac{|\widehat{\varphi}(\xi)|^{2}}{|\sin (\xi / 2)|} d \xi .
$$

The consistency of the scheme guarantees that $\psi_{\lambda}^{\prime}$ has at least one root in $[-\pi, \pi]$, namely at $\xi=0$. We consider $\widehat{\varphi}$ supported on $[0, \pi]$, the other case being similar. Let $I \subset[0, \pi]$ be the interval where $\psi_{\lambda}^{\prime}$ has the unique root $\xi=0$. We will prove that

$$
\sup _{j \in \mathbb{Z}} \sum_{n \geq 0}\left|\left(S_{\lambda}(n) \varphi\right)_{j}\right|^{2} \leq c(\lambda) \int_{I} \frac{|\widehat{\varphi}(\xi)|^{2}}{\left|\psi_{\lambda}^{\prime}(\xi)\right|} d \xi+c(\lambda) \int_{I^{c}}|\widehat{\varphi}(\xi)|^{2} d \xi .
$$

Taking into account that $\psi_{\lambda}^{\prime}(\xi) \sim-2 \lambda \xi$ as $\xi \sim 0$, we can replace $\left|\psi_{\lambda}^{\prime}(\xi)\right|$ by $|\sin (\xi / 2)|$ in (29) and thus (28).

Observing that $\psi_{\lambda}$ is one to one on $I$, we will prove that $V^{n}$ defined by

$$
V_{j}^{n}=\int_{I} e^{i j \xi} e^{i n \psi_{\lambda}(\xi)} m_{\lambda}^{n}(\xi) \widehat{\varphi}(\xi) d \xi, j \in \mathbb{Z}, n \geq 0,
$$

satisfies

$$
\sup _{j \in \mathbb{Z}} \sum_{n \geq 0}\left|V_{j}^{n}\right|^{2} \leq \int_{I} \frac{|\widehat{\varphi}(\xi)|^{2}}{\left|\psi_{\lambda}^{\prime}(\xi)\right|} d \xi .
$$

On $I^{c}=[0, \pi] \backslash I$ we will show that $W_{j}^{n}$ defined by

$$
W_{j}^{n}=\int_{I^{c}} e^{i j \xi} e^{i n \psi_{\lambda}(\xi)} m_{\lambda}^{n}(\xi) \widehat{\varphi}(\xi) d \xi
$$

satisfies

$$
\sup _{j \in \mathbb{Z}} \sum_{n \geq 0}\left|W_{j}^{n}\right|^{2} \leq \int_{I^{c}}|\widehat{\varphi}(\xi)|^{2} d \xi .
$$

We first prove (30). Using that $\psi_{\lambda}$ is one to one we can apply a change of variables and rewrite $V_{j}^{n}$ as

$$
V_{j}^{n}=\int_{\psi_{\lambda}(I)} e^{i n \xi} e^{i j \psi_{\lambda}^{-1}(\xi)} m_{\lambda}^{n}\left(\psi_{\lambda}^{-1}(\xi)\right) \widehat{\varphi}\left(\psi_{\lambda}^{-1}(\xi)\right)\left(\psi_{\lambda}^{-1}\right)^{\prime}(\xi) d \xi .
$$


Each of the above terms is similar to the Fourier coefficients of the function $\exp \left(i j \psi_{\lambda}^{-1}\right) \widehat{\varphi}\left(\psi_{\lambda}^{-1}\right)\left(\psi_{\lambda}^{-1}\right)^{\prime}$ except for the weight term $m_{\lambda}^{n}\left(\psi_{\lambda}^{-1}\right)$.

This is why we cannot apply Plancherel's identity, and thus we need to use the following Lemma:

Lemma 5.1. Let $m:[-\pi, \pi]$ be a continuous function satisfying

$$
0 \leq m(\xi) \leq 1, \xi \in[-\pi, \pi] .
$$

Then there exists a positive constant $c(m)$ such that the following inequality holds for any function $f \in L^{2}\left(\mathbb{T}^{1}\right)$

$$
\sum_{n \in \mathbb{Z}}\left|\int_{-\pi}^{\pi} e^{i n \xi} m^{|n|}(\xi) f(\xi) d \xi\right|^{2} \leq c(m) \int_{-\pi}^{\pi}|f(\xi)|^{2} d \xi,
$$

where $\mathbb{T}^{1}$ is the one-dimensional torus.

We postpone its proof. Applying Lemma 5.1 to $f=\exp \left(i j \psi_{\lambda}^{-1}\right) \widehat{\varphi}\left(\psi_{\lambda}^{-1}\right)\left(\psi_{\lambda}^{-1}\right)^{\prime}$ and to the multiplier $m_{\lambda}$ we obtain

$$
\begin{aligned}
\sum_{n \geq 0}\left|V_{j}^{n}\right| & \leq c(\lambda) \int_{\psi_{\lambda}(I)}\left|\widehat{\varphi}\left(\psi_{\lambda}^{-1}(\xi)\right)\right|^{2}\left|\left(\psi_{\lambda}^{-1}\right)^{\prime}(\xi)\right|^{2} d \xi \\
& =c(\lambda) \int_{\psi_{\lambda}(I)}\left|\widehat{\varphi}\left(\psi_{\lambda}^{-1}(\xi)\right)\right|^{2} \frac{d \xi}{\left|\psi_{\lambda}^{\prime}\left(\psi_{\lambda}^{-1}(\xi)\right)\right|^{2}}=c(\lambda) \int_{I} \frac{|\widehat{\varphi}(\xi)|^{2}}{\left|\psi_{\lambda}^{\prime}(\xi)\right|} d \xi
\end{aligned}
$$

where $c(\lambda)$ is independent of $j$.

We now proceed to prove (31). If $I^{c}$ is empty we have nothing to prove. Let us consider the case when $I^{c}$ is nonempty, i.e. it contains at least one root $\xi_{0}$ of $\psi_{\lambda}^{\prime}$. Without loss of generality we can assume that there is only one root. By our assumption (25), at the point $\xi_{0}$ we have that $m_{\lambda}\left(\xi_{0}\right)<1$. Let $J \subset I^{c}$ an interval containing $\xi_{0}$ such that $\sup _{J} m_{\lambda}=M_{\lambda}<1$. Let us define $J^{c}=I^{c} \backslash J$. On $J^{c}, \psi_{\lambda}^{\prime}$ has no zeros and thus a similar argument as in the proof of (30) shows that

$$
\sup _{j \in \mathbb{Z}} \sum_{n \geq 0}\left|\int_{J^{c}} e^{i j \xi} e^{i n \psi_{\lambda}(\xi)} m_{\lambda}^{n}(\xi) \widehat{\varphi}(\xi) d \xi\right|^{2} \leq c(\lambda) \int_{J^{c}} \frac{|\widehat{\varphi}(\xi)|^{2}}{\left|\psi_{\lambda}^{\prime}(\xi)\right|} d \xi \leq c(\lambda) \int_{J^{c}}|\widehat{\varphi}(\xi)|^{2} d \xi .
$$

It remains to prove that

$$
\sup _{j \in \mathbb{Z}} \sum_{n \geq 0}\left|\int_{J} e^{i j \xi} e^{i n \psi_{\lambda}(\xi)} m_{\lambda}^{n}(\xi) \widehat{\varphi}(\xi) d \xi\right|^{2} \leq \int_{J}|\widehat{\varphi}(\xi)|^{2} d \xi .
$$

Applying Cauchy's inequality to each term of the left hand side, we obtain that

$$
\sum_{n \geq 0}\left|\int_{J} e^{i j \xi} e^{i n \psi_{\lambda}(\xi)} m_{\lambda}^{n}(\xi) \widehat{\varphi}(\xi) d \xi\right|^{2} \leq \sum_{n \geq 0} M_{\lambda}^{2 n} \int_{J}|\widehat{\varphi}(\xi)|^{2} d \xi \leq c(\lambda) \int_{J}|\widehat{\varphi}(\xi)|^{2} d \xi
$$


which finishes the proof of inequality (26).

Step II. In the following we prove that if (25) fails then there is no constant $c(\lambda, s)$, independent of $h$, such that (11) holds for all $\varphi \in l^{2}(h \mathbb{Z})$. As in the case of lack of the integrability property the key point is the following Lemma:

Lemma 5.2. Let be $\xi_{0} \neq 0$ such that $\psi^{\prime}\left(\xi_{0}\right)=0$ and $m_{\lambda}\left(\xi_{0}\right)=1$. Then there exist two constants $c(\lambda)$ and $c_{1}(\lambda)$ such that for $N$ sufficiently large, there exists $\varphi_{N}$ such that $\left\|\varphi_{N}\right\|_{l^{2}(\mathbb{Z})} \simeq N^{1 / 2}$ and

$$
\left|\left(\left(-\Delta_{1}\right)^{s / 2} S_{\lambda}(n) \varphi\right)_{j}\right| \geq c(\lambda)
$$

for all $|j| \leq c_{1}(\lambda) N$ and $n \leq c_{1}(\lambda) N^{2}$.

We postpone the proof of this Lemma. Using the definition of $\left(-\Delta_{h}\right)^{s / 2}$ and that $k / h^{2}=\lambda$, we get

$$
\frac{k \sum_{n k \leq 1} h \sum_{|j| h \leq 1}\left|\left(\left(-\Delta_{h}\right)^{s / 2} S_{\lambda}(n) \varphi\right)_{j}\right|^{2}}{h \sum_{j \in \mathbb{Z}}\left|\varphi_{j}\right|^{2}}=\frac{\lambda h^{2-2 s} \sum_{n \leq 1 / \lambda h^{2}} \sum_{|j| \leq 1 / h}\left|\left(\left(-\Delta_{1}\right)^{s / 2} S_{\lambda}(n) \varphi\right)_{j}\right|^{2}}{\sum_{j \in \mathbb{Z}}\left|\varphi_{j}\right|^{2}} .
$$

Let us choose $N=1 / h$ and $\varphi_{N}$ as in Lemma 5.2. Then we obtain that

$$
\frac{k \sum_{n k \leq 1} h \sum_{|j| h \leq 1}\left|\left(D_{h}^{s} S_{\lambda}(n) \varphi\right)_{j}\right|^{2}}{h \sum_{j \in \mathbb{Z}}\left|\varphi_{j}\right|^{2}} \geq \frac{c(\lambda) N^{2 s-2} N^{3}}{N}=c(\lambda) N^{2 s},
$$

which finishes the proof.

We now prove the two Lemmas that we used before.

Proof. of Lemma 5.1. Let us define the linear operator $T f$ as:

$$
(T f)_{n}=\int_{-\pi}^{\pi} e^{i n \xi} m^{|n|}(\xi) f(\xi) d \xi, n \in \mathbb{Z} .
$$

Inequality (32) means that $T$ maps continuously $L^{2}(\mathbb{T})$ to $l^{2}(\mathbb{Z})$ or equivalently, its adjoint $T^{*}$ maps continuously $l^{2}(\mathbb{Z})$ to $L^{2}\left(\mathbb{T}^{1}\right)$. Explicit calculations show that $T^{*}$ has the following representation:

$$
\left(T^{*} g\right)(\xi)=\sum_{n \in \mathbb{Z}} e^{-i n \xi} m^{|n|}(\xi) g_{n}, \xi \in[-\pi, \pi] .
$$

It remains to prove that $T^{*}$ is well-defined and maps continuously $L^{2}(\mathbb{T})$ to $l^{2}(\mathbb{Z})$. The key point is the following pointwise estimate on $T^{*}$ :

$$
\left|\left(T^{*} g\right)(\xi)\right| \leq \sup _{0 \leq r \leq 1}\left|\sum_{n \in \mathbb{Z}} e^{i n \xi} r^{|n|} g_{n}\right|, \forall \xi \in[-\pi, \pi] .
$$


For any $0 \leq r \leq 1$, classical results on maximal functions (cf. Ref. [14], p. 76) show that

$$
\left|\sum_{n \in \mathbb{Z}} e^{i n \xi} r^{|n|} g_{n}\right| \leq M_{g^{\vee}}(\xi),
$$

where $g^{\vee}(\xi)=\sum_{n \in \mathbb{Z}} e^{i n \xi} g_{n}$ and $M_{f}$ is the maximal function of $f$, defined by

$$
M_{f}(t)=\sup _{0<s \leq \pi}\left|\frac{1}{2 s} \int_{t-s}^{t+s} f(\tau) d \tau\right| .
$$

Using that the maximal function $M_{g \vee}$ (cf. Ref. [14], p. 88) satisfies

$$
\left\|M_{g^{\vee}}\right\|_{L^{2}(\mathbb{T})} \leq\left\|g^{\vee}\right\|_{L^{2}(\mathbb{T})}=\|g\|_{l^{2}(\mathbb{Z})},
$$

we obtain in view of (33) and (34) that $T^{*}$ makes sense and maps continuously $L^{2}(\mathbb{T})$ to $l^{2}(\mathbb{Z})$.

Proof. of Lemma 5.2. Let us choose a function $\widehat{\varphi}_{1}$ supported in $(-1,1)$ with $\widehat{\varphi}_{1}>1$ on $(-1 / 2,1 / 2)$ and set for all $N \geq 1$

$$
\widehat{\varphi}_{N}(\xi)=N \widehat{\varphi}_{1}\left(N\left(\xi-\xi_{0}\right)\right) .
$$

Thus $\widehat{\varphi}_{N}$ is supported on $\left(\xi_{0}-N^{-1}, \xi_{0}+N^{-1}\right)$ and $\left\|\varphi_{N}\right\|_{l^{2}(\mathbb{Z})} \simeq N^{1 / 2}$. Applying mean value theorem and using that $|\sin (\xi / 2)| \sim|\xi / 2|$ on $[-\pi, \pi]$, we obtain that

$\left|\left(\left(-\Delta_{1}\right)^{s / 2} S_{\lambda}(n) \varphi_{N}\right)_{j}\right| \geq\left(1-2 N^{-1} \sup _{\xi \in \operatorname{supp} \widehat{\varphi}_{N}}\left|n \psi_{\lambda}^{\prime}(\xi)+j\right|\right) \int_{-\pi}^{\pi}|\xi|^{s} m_{\lambda}^{n}(\xi) \widehat{\varphi}_{N}(\xi) d \xi$.

Using that $\psi_{\lambda}^{\prime}$ vanishes at $\xi=\xi_{0}$ we get

$$
\left|n \psi_{\lambda}^{\prime}(\xi)+j\right| \leq|j|+n c_{\lambda}\left|\xi-\xi_{0}\right|, \xi \sim \xi_{0} .
$$

Then there exists a positive constant $c_{1}(\lambda)$ such that for all $j$ and $n$ satisfying $|j| \leq c_{1}(\lambda) N$ and $n \leq c_{1}(\lambda) N^{2}$ the following holds:

$$
2 N^{-1} \sup _{\xi \in \operatorname{supp} \widehat{\varphi}_{N}}\left|n \psi_{\lambda}^{\prime}(\xi)+j\right| \leq \frac{1}{2}
$$

and

$$
\left|\left(\left(-\Delta_{1}\right)^{s / 2} S_{\lambda}(n) \varphi_{N}\right)_{j}\right| \geq \frac{1}{2} \int_{-\pi}^{\pi}|\xi|^{s} m_{\lambda}^{n} \widehat{\varphi}_{N}(\xi) d \xi \geq \frac{\left|\xi_{0}\right|^{s} N}{2} \int_{\xi_{0}-N^{-1} / 2}^{\xi_{0}+N^{-1} / 2} m_{\lambda}^{n}(\xi) d \xi .
$$

The same arguments as in the proof of Lemma 4.1 show that the right hand term in the above estimate remains uniformly bounded by below by a positive constant $c(\lambda)$ for $N$ large enough. 


\section{Two examples}

Let us exemplify our previous work by considering two numerical schemes: the backward Euler scheme:

$$
i \frac{U_{j}^{n+1}-U_{j}^{n}}{k}+\frac{U_{j+1}^{n+1}-2 U_{j}^{n+1}+U_{j-1}^{n+1}}{h^{2}}=0, n \geq 0, j \in \mathbb{Z},
$$

and the Crank-Nicolson scheme:

$$
i \frac{U_{j}^{n+1}-U_{j}^{n}}{k}+\frac{U_{j+1}^{n+1}-2 U_{j}^{n+1}+U_{j-1}^{n+1}}{2 h^{2}}+\frac{U_{j+1}^{n}-2 U_{j}^{n}+U_{j-1}^{n}}{2 h^{2}}=0, n \geq 0, j \in \mathbb{Z} .
$$

For the first scheme the symbol $a_{\lambda}$ is given by:

$$
a_{\lambda}(\xi)=\frac{1}{1+4 i \lambda \sin ^{2} \frac{\xi}{2}}=\frac{\exp \left(-i \arctan \left(4 \lambda \sin ^{2} \frac{\xi}{2}\right)\right)}{\left(1+16 \lambda^{2} \sin ^{4} \frac{\xi}{2}\right)^{1 / 2}} .
$$

Explicit calculations show that the symbol $a_{\lambda}$ satisfies (13) and (25). In this case, even $\psi_{\lambda}$ vanishes its second derivatives at some points different from $\xi=0$, the dissipative character of $m_{\lambda}$ at these points allows us to recover the uniform decay property (14). More precisely the symbol $a_{\lambda}$ satisfies

$$
a_{\lambda}(\xi) \sim 1-i \lambda \xi^{2}-\lambda^{2} \xi^{4}+O\left(\xi^{6}\right), \xi \sim 0,
$$

which shows that the scheme is dissipative of order two:

$$
\left|a_{\lambda}(\xi)\right| \sim 1-\frac{\lambda \xi^{2}}{2}+O\left(\xi^{4}\right), \xi \sim 0 .
$$

In the case of the Crank-Nicolson scheme the symbol $a_{\lambda}$ is given by

$$
a_{\lambda}(\xi)=\frac{1-2 i \lambda \sin ^{2} \frac{\xi}{2}}{1+2 i \lambda \sin ^{2} \frac{\xi}{2}}=\exp \left(-2 i \arctan \left(2 \lambda \sin ^{2} \frac{\xi}{2}\right)\right) .
$$

Explicit calculations show that the derivative of the function $\psi_{\lambda}$ is given by

$$
\psi_{\lambda}^{\prime}(\xi)=\frac{-2 \lambda \sin \xi}{1+4 \lambda^{2} \sin ^{4} \frac{\xi}{2}}
$$

and the scheme fails to have property (25) at the point $\xi=\pi$. Also the second derivative of $\psi_{\lambda}$ satisfies $\psi_{\lambda}^{\prime \prime}(0) \psi_{\lambda}^{\prime \prime}(\pi / 2)<0$. This suffices to show that the scheme does not satisfy (13).

These pathologies are similar to the ones of the semidiscrete conservative scheme analyzed in Ref. [10] and additional techniques have to be introduced to cancel these spurious effects: filtering [9, numerical viscosity[10] or a twogrid preconditioner [1]. We point out that any filtration of initial data which excludes the end points $\xi= \pm \pi$ will guarantee the local smoothing property (11). Regarding the $l^{1}(h \mathbb{Z})-l^{\infty}(h \mathbb{Z})$ decay, there exist two points $\pm \xi_{0} \in[-\pi, \pi]$ where the second derivative of $\psi_{1}$ vanishes. Any filtration of initial data which excludes these two points will recover the right decay property of solutions. 


\section{Strichartz like estimates}

In this section we consider a numerical scheme which obeys the condition (13). For such a scheme we prove time-space estimates similar to the Strichartz estimates in the continuous case. These estimates are important in the further analysis of the approximations of nonlinear problems. We recall that in the continuous case the Strichartz estimates play a crucial role in proving the wellposedness of the nonlinear Schrödinger equations for a class of nonlinearities that cannot be treated by energy arguments.

Theorem 7.1. Let $A_{\lambda}$ be such that its symbol $a_{\lambda}$ satisfies (13) and $(q, r)$ and $(\tilde{q}, \tilde{r})$ be two admissible pairs. Then

i) There exists a positive constant $C(\lambda, r)$ such that

$$
\left\|S_{\lambda}(\cdot) \varphi\right\|_{l^{q}\left(k \mathbb{N}, l^{r}(h \mathbb{Z})\right)} \leq C(\lambda, r)\|\varphi\|_{l^{2}(h \mathbb{Z})}
$$

holds for all $\varphi \in l^{2}(h \mathbb{Z})$, uniformly on $h>0$.

ii) There exists a positive constant $C(\lambda, r)$ such that

$$
\left\|\sum_{n \geq 0} S_{\lambda}(n)^{*} f(n)\right\|_{l^{2}(h \mathbb{Z})} \leq C(\lambda, r)\|f\|_{l^{q^{\prime}}\left(k \mathbb{N}, l^{r^{\prime}}(h \mathbb{Z})\right)}
$$

holds for all $f \in l^{q^{\prime}}\left(k \mathbb{N}, l^{r^{\prime}}(h \mathbb{Z})\right)$, uniformly on $h>0$.

iii) There exists a positive constant $C(\lambda, r, \tilde{r})$ such that

$$
\|\Lambda f\|_{l^{q}\left(k \mathbb{N}, l^{r}(h \mathbb{Z})\right)} \leq C(\lambda, q, r, \tilde{q}, \tilde{r})\|f\|_{l_{\tilde{q}^{\prime}}\left(k \mathbb{N}, l^{\tilde{r}^{\prime}}(h \mathbb{Z})\right)}
$$

holds for all $f \in l^{\tilde{q}^{\prime}}\left(k \mathbb{N}, l^{\tilde{r}^{\prime}}(h \mathbb{Z})\right)$, uniformly on $h>0$.

Remark 7.1. In the particular case when for some positive integer $N, f(n)$ vanishes identically for all $n>N$ then (39) implies that the following also holds:

$$
\left\|\sum_{n=0}^{N} S_{\lambda}(n)^{*} f(n)\right\|_{l^{2}(h \mathbb{Z})} \leq C(\lambda, r)\left[\sum_{n=0}^{N} k\|f(n)\|_{l^{r^{\prime}}(h \mathbb{Z})}^{q}\right]^{1 / q}
$$

with a constant $C(\lambda, q, r)$ independent on $N$ and $h$. This estimate will be useful in the proof of $(40)$.

Proof. A scaling argument as in the previous proofs shows that it is sufficient to consider the case $h=1$.

By duality the proof of (38) is reduced to the one of (39). Also, inequality (39) turns out to be equivalent to the bilinear estimate

$$
\left|\left\langle\sum_{n \geq 0} S_{\lambda}(n)^{*} f(n), \sum_{m \geq 0} S_{\lambda}(m)^{*} g(m)\right\rangle\right| \leq C(\lambda, r)\|f\|_{l^{q^{\prime}}\left(\mathbb{N}, l^{r^{\prime}}(\mathbb{Z})\right)}\|g\|_{l^{q^{\prime}}\left(\mathbb{N}, l^{r^{\prime}}(\mathbb{Z})\right)},
$$


where $\langle\cdot, \cdot\rangle$ is the $l^{2}(\mathbb{Z})$-inner product. In fact we prove the stronger inequality:

$$
\sum_{n \geq 0} \sum_{m \geq 0}\left|\left\langle S_{\lambda}(n)^{*} f(n), S_{\lambda}(m)^{*} g(m)\right\rangle\right| \leq C(\lambda, r)\|f\|_{l^{\prime}\left(\mathbb{N}, l^{r^{\prime}}(\mathbb{Z})\right)}\|g\|_{l^{q^{\prime}}\left(\mathbb{N}, l^{\prime}(\mathbb{Z})\right)} .
$$

In view of estimate (16) we have

$$
\begin{aligned}
\left|\left\langle S_{\lambda}(n)^{*} f(n), S_{\lambda}(m)^{*} g(m)\right\rangle\right| & =\left|\left\langle f(n), S_{\lambda}(n) S_{\lambda}(m)^{*} g(m)\right\rangle\right| \\
& \leq\|f(n)\|_{l^{r^{\prime}}(\mathbb{Z})}\left\|S_{\lambda}(n) S_{\lambda}(m)^{*} g(m)\right\|_{l^{r}(\mathbb{Z})} \\
& \leq C(\lambda, r)\|f(n)\|_{l^{r^{\prime}}(\mathbb{Z})} \frac{\|g(m)\|_{l^{r^{\prime}}(\mathbb{Z})}}{1+|n-m|^{2 / q}} .
\end{aligned}
$$

At this point we make use of the following Lemma 19, which is a discrete version of the well-known Hardy-Litlewood-Sobolev inequality (cf. Ref. 22, p. 119):

Lemma 7.1. Let be $0<\alpha<1$ and $k$ a sequence such that

$$
|k(n)| \leq \frac{1}{1+|n|^{\alpha}}, \forall n \in \mathbb{Z} .
$$

Then the operator $\mathcal{T}$ defined by $\mathcal{T}(f)=f * k$ maps continuously $l^{p}(\mathbb{Z})$ into $l^{q}(\mathbb{Z})$ for any $p$ and $q$ satisfying

$$
1<p<q<\infty \text { and } \frac{1}{q}=\frac{1}{p}-1+\alpha .
$$

In view of this Lemma and applying Hölder's inequality in variable $n$ we obtain that

$$
\begin{aligned}
\sum_{n \geq 0} \sum_{m \geq 0} \mid\left\langle S_{\lambda}(n)^{*} f(n),\right. & \left.S_{\lambda}(m)^{*} g(m)\right\rangle \mid \leq C(\lambda, r) \sum_{n \geq 0} \sum_{m \geq 0}\|f(n)\|_{l^{r^{\prime}}(\mathbb{Z})} \frac{\|g(m)\|_{l^{r^{\prime}}(\mathbb{Z})}}{1+|n-m|^{2 / q}} \\
& \leq C(\lambda, r)\|f\|_{l^{\prime}\left(\mathbb{N}, l^{\prime}(\mathbb{Z})\right)}\left\|\left(\sum_{m \geq 0} \frac{\|g(m)\|_{l^{r^{\prime}}(\mathbb{Z})}}{1+|n-m|^{2 / q}}\right)\right\|_{l^{q}(\mathbb{Z})} \\
& \leq C(\lambda, r)\|f\|_{l^{\prime}\left(\mathbb{N}, l^{r^{\prime}}(\mathbb{Z})\right)}\|g\|_{l^{\prime}\left(\mathbb{N}, l^{\prime}(\mathbb{Z})\right)}
\end{aligned}
$$

and then (39).

It remains to prove inequality (40). We consider the cases $(\tilde{q}, \tilde{r})=(\infty, 2)$, $(q, r)=(\infty, 2)$ and $(\tilde{q}, \tilde{r})=(q, r)$, since the other cases follow by interpolation. By duality

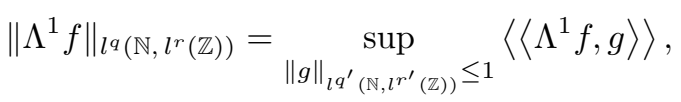

where $\langle\langle\cdot, \cdot\rangle\rangle$ is the inner product on $l^{2}\left(\mathbb{N}, l^{2}(\mathbb{Z})\right)$. 
Let us choose a function $g$ in $l^{q^{\prime}}\left(\mathbb{N}, l^{r^{\prime}}(\mathbb{Z})\right)$. The definition of $\Lambda^{1}$ gives us

$$
\begin{aligned}
\left\langle\left\langle\Lambda^{1} f, g\right\rangle\right\rangle & =\sum_{n \geq 0}\left\langle\sum_{j=0}^{n} S_{\lambda}(n-j) f(j), g(n)\right\rangle \\
& =\sum_{j \geq 0}\left\langle f(j), \sum_{n \geq j} S_{\lambda}(n-j)^{*} g(n)\right\rangle .
\end{aligned}
$$

In the case $(\tilde{q}, \tilde{r})=(\infty, 2)$, Cauchy's inequality applied to (42) shows that

$$
\begin{aligned}
\left\langle\left\langle\Lambda^{1} f, g\right\rangle\right\rangle & \leq \sum_{j \geq 0}\|f(j)\|_{l^{2}(\mathbb{Z})}\left\|\sum_{n \geq j} S_{\lambda}(n-j)^{*} g(n)\right\|_{l^{2}(\mathbb{Z})} \\
& \leq\|f\|_{l^{1}\left(\mathbb{N}, l^{2}(\mathbb{Z})\right)} \sup _{j \geq 0}\left\|\sum_{m \geq 0} S_{\lambda}(m)^{*} g(m+j)\right\|_{l^{2}(\mathbb{Z})} .
\end{aligned}
$$

Applying estimate (39) to the function $g(\cdot+j)$ we obtain the desired inequality for $(\tilde{q}, \tilde{r})=(\infty, 2)$.

In view of (42), we also obtain that

$$
\left\langle\left\langle\Lambda^{1} f, g\right\rangle\right\rangle \leq\left\|\sum_{0 \leq j \leq n} S_{\lambda}(n-j) f(j)\right\|_{l^{\infty}\left(\mathbb{N}, l^{2}(\mathbb{Z})\right)}\|g\|_{l^{1}\left(\mathbb{N}, l^{2}(\mathbb{Z})\right)} .
$$

We write

$$
\sum_{j=0}^{n} S_{\lambda}(n-j) f(j)=\sum_{j=0}^{n} \overline{S_{\lambda}(n-j)^{*} \overline{f(j)}}=\sum_{j=0}^{n} \overline{S_{\lambda}(j) * \overline{f(n-j)}}
$$

and apply estimate (41) to the function $f(n-\cdot)$.

It remains to analyze the case $(q, r)=(\tilde{q}, \tilde{r})$. Observe that $\Lambda^{1}$ satisfies for any $n \geq 0$ the rough estimate

$$
\left\|\left(\Lambda^{1} f\right)(n)\right\|_{l^{r}(\mathbb{Z})} \leq \sum_{j=0}^{n}\left\|S_{\lambda}(n-j) f(j)\right\|_{l^{r}(\mathbb{Z})} \leq C(\lambda, r) \sum_{0 \leq j \leq n} \frac{\|f(j)\|_{l^{r^{\prime}}(\mathbb{Z})}}{1+|n-j|^{2 / q}}
$$

The same arguments as in the proof of (39) based on Lemma 7.1, show that

$$
\left\|\Lambda^{1} f\right\|_{l^{q}\left(\mathbb{N}, l^{r}(\mathbb{Z})\right)} \leq C(\lambda, r)\|f\|_{l^{q^{\prime}}\left(\mathbb{N}, l^{r^{\prime}}(\mathbb{Z})\right)},
$$

which finishes the proof of the last case and that of Theorem 7.1 .

\section{Application to a nonlinear problem}

In this section we consider a numerical scheme for the semilinear NSE equation in $\mathbb{R}$ with repulsive power law nonlinearity :

$$
\left\{\begin{array}{cl}
i u_{t}+u_{x x} & =|u|^{p} u, \quad x \in \mathbb{R}, t>0 \\
u(0, x) & =\varphi(x), \quad x \in \mathbb{R}
\end{array}\right.
$$


$p<4$ and initial data in $L^{2}(\mathbb{R})$. The case of nonlinearity $f(u)=-|u|^{p} u$ could be treated in the same manner. In fact, the key point in the global existence of the solutions is that the $L^{2}$-scalar product $(f(u), u)$ is a real number. All the results presented here extend to more general nonlinearities [3] $f(u)$. The critical case $p=4$ could be also treated by imposing smallness on the initial data.

With the notation $f(x)=|x|^{p} x$ the scheme we propose is given by

$$
\left\{\begin{array}{l}
i \frac{U_{j}^{n+1}-U_{j}^{n}}{k}+\frac{U_{j+1}^{n+1}-2 U_{j}^{n+1}+U_{j}^{n+1}}{h^{2}}=f\left(U_{j}^{n+1}\right), n \geq 0, j \in \mathbb{Z}, \\
U_{j}^{0}=\varphi_{j}, j \in \mathbb{Z}
\end{array}\right.
$$

$\varphi \in l^{2}(h \mathbb{Z})$ being an approximation of the initial datum $\varphi$ and $h$ and $k$ such that $k / h^{2}$ remains constant. This implies the existence of two operators $A_{1, \lambda}$ and $A_{2, \lambda}$ such that

$$
U^{n+1}=\left(A_{1, \lambda}^{-1}\right) A_{2, \lambda} U^{n}+k A_{1, \lambda}^{-1} f\left(U^{n+1}\right), n \geq 0 .
$$

Using the notation $S_{\lambda}(n) \varphi$ for the solution at the step $n$ of the backward Euler scheme for the linear Schrödinger equation, the solution of the above equation is also solution of the following one:

$$
U^{n}=S_{\lambda}(n) \varphi+k \sum_{j=1}^{n} S_{\lambda}(n-j) A_{1, \lambda}^{-1} f\left(U^{j}\right) .
$$

Concerning the existence of solutions for problem (8) the main result is given by the following Theorem.

Theorem 8.1. Let $p \in[0,4)$ and $U^{0} \in l^{2}(h \mathbb{Z})$. Then there is a unique solution of equation (44) which satisfies:

$$
\left\|U^{n}\right\|_{l^{2}(h \mathbb{Z})} \leq\left\|U^{0}\right\|_{l^{2}(h \mathbb{Z})}
$$

for all $n \geq 0$.

Moreover, for all $T>0$ and $(q, r)$ an admissible pair there is a constant $C(T, r)$ such that

$$
\|U\|_{l^{q}\left(n k \leq T, l^{r}(h \mathbb{Z})\right)} \leq C(T, r)\left\|U^{0}\right\|_{l^{2}(h \mathbb{Z})}
$$

uniformly on $h$.

Proof. The proof consists in applying the Banach fix point Theorem in a ball of $l^{q}\left(n k \leq T, l^{r}(h \mathbb{Z})\right) \cap L^{\infty}\left(n k \leq T, l^{2}(h \mathbb{Z})\right)$ and in making use of the Strichartzlike estimates proved in Theorem 7.1. Observe that the nonlinear term $f(U)$ is composed by the operator $\left(A_{1, \lambda}\right)^{-1}$. In order to apply the Banach fix point Theorem we have to prove that the operator $\left(A_{1, \lambda}\right)^{-1}$ is continuous from $l^{s}(h \mathbb{Z})$ to $l^{s}(h \mathbb{Z})$ for any $s \in[1,2]$, with a norm independent of $h>0$. Observe that by scaling it is sufficient to prove that

$$
\left\|\left(A_{1, \lambda}\right)^{-1} f\right\|_{l^{s}(\mathbb{Z})} \leq c(\lambda)\|f\|_{l^{s}(\mathbb{Z})}
$$


for all $f \in l^{s}(\mathbb{Z})$. Using the kernel representation of $\left(A_{1, \lambda}\right)^{-1} f$ in convolution form

$$
\left(A_{1, \lambda}\right)^{-1} f=K_{\lambda} * f
$$

where $K_{\lambda}$ is given by

$$
\widehat{K}_{\lambda}(\xi)=\frac{1}{1+\lambda \sin ^{2}\left(\frac{\xi}{2}\right)}, \xi \in[-\pi, \pi],
$$

it is sufficient to show that $\left\|K_{\lambda}\right\|_{l^{1}(\mathbb{Z})} \leq c(\lambda)$. Using Carlson-Beurling inequality [2] we get

$$
\left\|K_{\lambda}\right\|_{l^{1}(\mathbb{Z})} \leq\left(\left\|\widehat{K}_{\lambda}\right\|_{L^{2}((-\pi, \pi))}\left\|\left(\widehat{K}_{\lambda}\right)^{\prime}\right\|_{L^{2}((-\pi, \pi))}\right)^{1 / 2}=c(\lambda)
$$

This allows us to prove the local existence of the solution of Eq. (44) and estimate (46).

To guarantee the global existence of the solution we prove a priori estimates on the $l^{2}$-norm of the solutions. Multiplying equation (44) by $\bar{U}_{j}^{n+1}$ we get for all $n \geq 0$ and $j \in \mathbb{Z}$ :

$$
i\left|U_{j}^{n+1}\right|^{2}-i U_{j}^{n} \bar{U}_{j}^{n+1}+\lambda\left(U_{j+1}^{n+1}-2 U_{j}^{n}+U_{j-1}^{n+1}\right) \bar{U}_{j}^{n+1}=k f\left(U_{j}^{n+1}\right) \bar{U}_{j}^{n+1} .
$$

Summing up on $j \in \mathbb{Z}$ and taking the imaginary part we obtain

$$
\sum_{j \in \mathbb{Z}}\left|U_{j}^{n+1}\right|^{2} \leq \sum_{j \in \mathbb{Z}}\left|U_{j}^{n+1} U_{j}^{n}\right|,
$$

which guarantees that the $l^{2}$-norm of $U^{n}$ is bounded above by the $l^{2}$-norm of the initial datum. This guarantees the $l^{2}$-stability of the scheme and the global existence of a solution $\left(U^{n}\right)_{n \geq 0}$.

\section{Convergence of the method}

In what follows we introduce the interpolator $I^{h} U$, piecewise linear in time and space.

Theorem 9.1. Let $p<4$ and $k$ and $h$ be such that the Courant number $k / h^{2}$ is a kept constant. Then the interpolator $I^{h} U$ satisfies

$$
\left\|I^{h} U\right\|_{L^{\infty}\left([0, \infty), L^{2}(\mathbb{R})\right)} \leq\left\|\left(I^{h} U\right)(0)\right\|_{L^{2}(\mathbb{R})} .
$$

and for all $T>0$ and $(q, r)$ an admissible pair there is a positive constant $C(T)$ such that

$$
\left\|I^{h} U\right\|_{L^{q}\left([0, T], L^{r}(\mathbb{R})\right)} \leq C(T)\left\|\left(I^{h} U\right)(0)\right\|_{L^{2}(\mathbb{R})} .
$$

Moreover

$$
I^{h} U \stackrel{*}{\rightarrow} u \quad \text { in } \quad L^{\infty}\left([0, \infty), L^{2}(\mathbb{R})\right),
$$




$$
I^{h} U \rightarrow u \quad \text { in } \quad L_{\text {loc }}^{q}\left([0, \infty), L^{r}(\mathbb{R})\right),
$$

and

$$
I^{h} U \rightarrow u \text { a.e. on }[0, \infty) \times \mathbb{R},
$$

where $u$ is the unique weak solution of the NSE.

Proof. The first two estimates are a consequence of (45) and (46). Thus, obviously (47) and (48) hold. The limit (49) is a consequence of the local smoothing property of the discrete operator $S_{\lambda}$ that allows us to prove the uniform boundedness of solutions in $L_{l o c}^{2}\left(\mathbb{R}, H_{\text {loc }}^{s}(\mathbb{R})\right)$, for some positive constant $s$, and thus compactness for the sequence $\left\{I^{h} U\right\}_{h>0}$. All the above properties show the convergence of $I^{h} u$ towards the unique solution $u$ of the NSE.

\section{A finer analysis of the Crank-Nicolson Scheme}

In this section we analyze whether the two-grid pre-conditioner, introduced by Glowinsky $[8$, recovers the dispersive properties (14) of the Crank-Nicolson scheme. The two-grid method is roughly as follows. Two meshes are considered: the coarse one of size $p h, p \geq 1$ integer, $p h \mathbb{Z}$, and the fine one, $h \mathbb{Z}$, of size $h>0$. The method relies basically on solving the finite-difference discretization (37) on the fine mesh $h \mathbb{Z}$, but only for slow data, interpolated from the coarse grid $p h \mathbb{Z}$. This method with $p=4$ has been used successfully in Ref. 11] to prove uniform dispersive estimates for conservative semidiscrete approximations of the Schrödinger equation.

A careful Fourier analysis of initial data obtained by the above algorithm, shows that their discrete Fourier transform is modulated by a multiplier $q_{p}$ which vanishes quadratically at the points $\xi= \pm \pi r / p, 1 \leq r \leq p-1$ of the one dimensional torus $\mathbb{T}^{1}$.

In the case of a semidiscrete approximation of the one dimensional linear Schrödinger equation the symbol introduced by the scheme is $\psi(\xi)=\sin ^{2}(\xi / 2)$ and vanishes its second derivative at $\pm \pi / 2$. As proved in Ref. [1], a twoalgorithm with quotient of the meshes $1 / 4$ cancels the spurious effects at the points $\pm \pi / 2$, and the scheme has uniform dispersive properties in that class of data.

The condition that the multiplicative factor $q_{p}$ vanishes at the roots $\xi_{0} \neq 0$ of $\psi_{\lambda}^{\prime \prime}$ is necessary. If not, using that the multiplicative factor $q_{p}$ behaves as a nonzero constant near the point $\xi_{0}$, we can choose initial data concentrated at this point as we already did in the proof of (17) and the dispersive properties fail to be uniform on the mesh size.

In the following we prove that for any Courant number $\lambda=k / h^{2} \in \mathbb{Q}$, there is no two-grid pre-conditioner that guarantees the dispersive properties (14) in this particular class of data. We prove that any two-grid algorithm introduces a multiplicative factor that vanishes only at points belonging to $2 \pi \mathbb{Q}$. On the other hand, for any rational Courant number $\lambda$, the second derivative of the symbol $\psi_{\lambda}$ introduced by the Crank-Nicolson scheme vanishes at some point that does not belong to the set $2 \pi \mathbb{Q}$, and thus, similar to (17) estimate holds. 
For the local smoothing properties (26), a two-grid pre-conditioner with $p=2$, allows us to recover that property. The essential point is that the first derivative of $\psi_{\lambda}$ vanishes at the points $\pm \pi$ and a two-grid algorithm with the quotient of the meshes $1 / 2$ will vanish the spurious effects at these points.

The following lemma gives a characterization of data that are obtained by a two-grid algorithm involving the meshes $p \mathbb{Z}$ and $\mathbb{Z}$. Its proof uses only the definition of the discrete Fourier transform and for that we omit it.

Lemma 10.1. Let $p \geq 2$ and $\{V(p k)\}_{k \in \mathbb{Z}}$ a function defined on the coarse grid

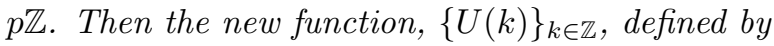

$$
U(k p+j)=\frac{(p-j) V(k p)+j V((k+1) p)}{p}, k \in \mathbb{Z}, j=0, \ldots, p-1,
$$

satisfies

$$
\widehat{U}(\xi)=\frac{e^{i(p-1) \xi} \widehat{V}(\xi)}{p}\left(\sum_{k=0}^{p-1} e^{i k \xi}\right)^{2}, \xi \in[-\pi, \pi] .
$$

Remark 10.1. We point out that the multiplicative factor $\sum_{k=0}^{p-1} e^{i k \xi}$ vanishes only at the points $\xi_{k}=2 k \pi / p$ with $k=1, \ldots, p-1$.

The symbol $a_{\lambda}$ of Crank-Nicolson's scheme is given by $a_{\lambda}(\xi)=\exp \left(i \psi_{\lambda}(\xi)\right)$ where

$$
\psi_{\lambda}(\xi)=2 \arctan \left(2 \lambda \sin ^{2} \frac{\xi}{2}\right)
$$

The first two derivatives of $\psi_{\lambda}$ are given by

$$
\psi_{\lambda}^{\prime}(\xi)=\frac{-2 \lambda \sin \xi}{1+4 \lambda^{2} \sin ^{4} \frac{\xi}{2}}, \quad \xi \in[-\pi, \pi]
$$

and

$$
\psi_{\lambda}^{\prime \prime}(\xi)=\frac{-2 \lambda\left(\cos \xi-\lambda^{2}(1-\cos \xi)^{2}(2+\cos \xi)\right)}{\left(1+4 \lambda^{2} \sin ^{4} \frac{\xi}{2}\right)^{2}}, \quad \xi \in[-\pi, \pi] .
$$

We prove that for any $\lambda \in \mathbb{Q}$, the function

$$
g_{\lambda}(\xi)=\cos \xi-\lambda^{2}(1-\cos \xi)^{2}(2+\cos \xi)
$$

has at least one root that does not belong to $2 \pi \mathbb{Q}$. The existence of a root easily follows: $g_{\lambda}(0) g_{\lambda}(\pi / 2)<0$.

Let us suppose the existence of $\lambda \in \mathbb{Q}$ such that the function $g_{\lambda}$ has a root of the form $2 \pi m / n$ with $m, n \in \mathbb{Z},(m, n)=1$. We write $\cos \xi=\left(e^{i \xi}+e^{-i \xi}\right) / 2$ and set $\mu=\lambda^{2} / 4$ in equation (50). This gives us that $\xi$ satisfies the following equation

$$
e^{i \xi}+e^{-i \xi}-\mu\left(2-e^{-i \xi}-e^{i \xi}\right)^{2}\left(4+e^{i \xi}+e^{-i \xi}\right)=0 .
$$

Then the polynomial $P_{\mu}(x)$, defined by

$$
P_{\mu}(x)=x^{4}+x^{2}-\mu\left(x^{2}-2 x+1\right)^{2}\left(x^{2}+4 x+1\right)
$$


admits a root of the form $x=\exp (2 i \pi m / n)$, with $(m, n)=1$. This implies that $P_{\mu}(x)$ is divisible by some cyclotomic polynomials associated with the root $2 \pi m / n$. Using the fact that the degree of the cyclotomic polynomial of order $n, Q_{n}$, is $\varphi(n)$ the Euler $\varphi$-function, we obtain that $n$ satisfies $\varphi(n) \leq 6$. The possible values of $n$ belong to the set $\{1,2,3,5,6,7,9,12\}$. In order to obtain a contradiction it remains to prove that none of the following polynomials $Q_{n}$ divides $P_{\mu}$ :

$$
\begin{array}{ll}
Q_{1}=x-1, & Q_{2}=x+1 \\
Q_{3}=x^{2}+x+1, & Q_{5}=x^{4}+x^{3}+x^{2}+x+1, \\
Q_{6}=x^{2}-x+1, & Q_{7}=x^{6}+x^{5}+x^{4}+x^{3}+x^{2}+x+1, \\
Q_{9}=x^{6}+x^{3}+1, & Q_{12}=x^{4}-x^{2}+1 .
\end{array}
$$

where $Q_{n}$ is the cyclotomic polynomial of order $n$. Explicit calculations show that

$$
P_{\mu} \equiv 2 \bmod Q_{1}, \quad P_{\mu} \equiv 2+2^{5} \mu \bmod Q_{2}
$$

and

$$
P_{\mu} \equiv-1-27 \mu^{2} \quad \bmod Q_{3}, \quad P_{\mu} \equiv-1+5 \mu^{2} \quad \bmod Q_{6}
$$

which exclude the cases $Q_{1}, Q_{2}, Q_{3}, Q_{6}$. In the case of $Q_{5}$ we get

$$
P_{\mu} \equiv-x^{3}\left(25 \mu^{2}+1\right)+\ldots \quad \bmod Q_{5}
$$

which proves that $Q_{5} \backslash P_{\mu}$. Similar calculations show that

$$
P_{\mu} \equiv x^{4}\left(9 \mu^{2}+1\right)+\ldots \quad \bmod Q_{9}, \quad P_{\mu} \equiv-15 \mu^{2} x^{3} \quad \bmod Q_{12} .
$$

It remains to study the case of $Q_{7}$. Using that both polynomials have the same degree, $P_{\mu}$ equals $Q_{7}$ multiplied by a constant. Using the fact that the coefficient of $x$ in $P_{\mu}$ vanishes, we also exclude this case.

\section{Acknowledgment}

The author wishes to thank the guidance of his Ph.D advisor Enrique Zuazua.

This work has been supported by the doctoral fellowship AP2003-2299 of MEC (Spain) and the grants MTM2005-00714 and DOMINO (CIT-3702002005-10) of the MEC (Spain), 80/2006 of CNCSIS (Romania).

\section{References}

[1] K. Atkinson and W. Han, Theoretical numerical analysis. A functional analysis framework, Texts in Applied Mathematics, Vol. 39 (SpringerVerlag, 2005).

[2] P. Brenner and V. Thomée, Stability and convergence rates in $L_{p}$ for certain difference schemes, Math. Scand. 27 (1970) 5-23. 
[3] T. Cazenave, Semilinear Schrödinger equations, Courant Lecture Notes in Mathematics, Vol. 10 (American Mathematical Society 2003).

[4] T. Cazenave and F.B. Weissler, The Cauchy problem for the nonlinear Schrödinger equation in $H^{1}$, Manuscripta Math. 61 (1988) 477-494.

[5] T. Cazenave and F.B. Weissler, Some remarks on the nonlinear Schrödinger equation in the critical case, in Nonlinear semigroups, partial differential equations and attractors, Lecture Notes in Math., Vol. 1394, (Springer, 1989) pp. 18-29.

[6] P. Constantin and J.C. Saut, Local smoothing properties of Schrödinger equations, Indiana Univ. Math. J. 38 (1989) 791-810.

[7] J. Ginibre and G. Velo, The global Cauchy problem for the nonlinear Schrödinger equation revisited, Ann. Inst. H. Poincaré Anal. Non Linéaire 2 (1985) 309-327.

[8] R. Glowinski, Ensuring well-posedness by analogy; Stokes problem and boundary control for the wave equation, J. Comput. Phys. 103 (1992) $189-221$.

[9] L.I. Ignat, Propiedades cualitativas de esquemas numéricos de aproximación de ecuaciones de difusión y de dispersión, Ph.D thesis, Universidad Autónoma de Madrid, 2006.

[10] L.I. Ignat and E. Zuazua, Dispersive properties of a viscous numerical scheme for the Schrödinger equation, C. R. Acad. Sci. Paris, Ser. I 340 (2005) 529-534.

[11] L.I. Ignat and E. Zuazua, A two-grid approximation scheme for nonlinear Schrödinger equations: dispersive properties and convergence, C. R. Acad. Sci. Paris, Ser. I 341 (2005) 381-386.

[12] L.I. Ignat and E. Zuazua, Dispersive properties of numerical schemes for nonlinear Schrödinger equations, in Foundations of Computational Mathematics, Santander 2005, London Mathematical Society lecture Notes, Vol. 331, L. M. Pardo et al. eds, (Cambridge Univ. Press 2006) pp. 181-207.

[13] A. Iserles, A first course in the numerical analysis of differential equations, (Cambridge University Press 1996).

[14] Y. Katznelson, An introduction to harmonic analysis, (Cambridge University Press), 2004).

[15] M. Keel and T. Tao, Endpoint Strichartz estimates, Am. J. Math. 120 (1998) 955-980.

[16] C.E. Kenig, G. Ponce and L. Vega, Oscillatory integrals and regularity of dispersive equations, Indiana Univ. Math. J. 40 (1991) 33-69. 
[17] C.E. Kenig, G. Ponce and L. Vega, Small solutions to nonlinear Schrödinger equations, Ann. Inst. H. Poincaré Anal. Non Linéaire 10 (1993) 255-288.

[18] A. Magyar, E. M. Stein and S. Wainger, Discrete analogues in harmonic analysis: spherical averages, Ann. of Math. 155 (2002) 189-208.

[19] M. Nixon, The discretized generalized Korteweg-de Vries equation with fourth order nonlinearity, J. Comput. Anal. Appl. 5 (2003) 369-397.

[20] M. Plancherel and G. Pólya, Fonctions entières et intégrales de Fourier multiples. II, Comment. Math. Helv. 10 (1937), 110-163.

[21] A. Stefanov and P.G. Kevrekidis, Asymptotic behaviour of small solutions for the discrete nonlinear Schrödinger and Klein-Gordon equations, Nonlinearity 18 (2005) 1841-1857.

[22] E.M. Stein, Singular integrals and differentiability properties of functions, (Princeton University Press, 1973).

[23] E.M. Stein, Harmonic analysis: Real-variable methods, orthogonality, and oscillatory integrals, (Princeton University Press, 1993).

[24] R.S. Strichartz, Restrictions of Fourier transforms to quadratic surfaces and decay of solutions of wave equations, Duke Math. J. 44 (1977) 705-714.

[25] C. Sulem and P.L. Sulem, The nonlinear Schrödinger equation. Selffocusing and wave collapse, Applied Mathematical Sciences, Vol. 139, (Springer-Verlag 1999).

[26] Y. Tsutsumi, $L^{2}$-solutions for nonlinear Schrödinger equations and nonlinear groups, Funkc. Ekvacioj Ser. Int. 30 (1987) 115-125.

[27] L. Vega, Schrödinger equations: pointwise convergence to the initial data, Proc. Amer. Math. Soc. 102 (1988) 874-878.

[28] K. Yajima, Existence of solutions for Schrödinger evolution equations, Comm. Math. Phys. 110 (1987) 415-426. 